\title{
Modeling and Simulation of Transient Impact Behavior of Elastic-Plastic Beam
}

\author{
Taoufik Kriflou ${ }^{1, *}$, Mohamed Rachik ${ }^{2}$, Lahcen Azrar, ${ }^{3,4}$ Khalid EL Bikri ${ }^{1}$ \\ ${ }^{1}$ Research Center STIS, M2SM, Mechanical Engineering Department, ENSET, Mohammed V University, Morocco \\ ${ }^{2}$ Laboratoire Roberval, FRE CNRS-UTC 2012, Sorbonne université, Université de Technologie de Compiègne, Centre de recherche \\ Royallieu, CS60319, 60203, Compiègne, France \\ ${ }^{3}$ Research Center STIS, M2CM, Department of Applied Mathematics and Informatics, ENSET, Mohammed V University, Morocco \\ ${ }^{4}$ Department of Mechanical Engineering, Faculty of Engineering, KAU, Jeddah, Saudi Arabia
}

Received June 16, 2019; Revised October 7, 2019; Accepted October 16,2019

Copyright $\mathrm{C} 2019$ by authors, all rights reserved. Authors agree that this article remains permanently open access under the terms of the Creative Commons Attribution License 4.0 International License

\begin{abstract}
The transverse spherical impact on an elastic-plastic beam is formulated and investigated herein. Both semi-analytical procedure and finite element (FEM) solution are elaborated. The semi analytical solution combines a finite difference method with the Hertz contact theory. The transient response of impact beams is computed by considering the loaded and unloaded phases. The contact force calculation is based on the model proposed by Stronge. To validate our semi-analytical model, a 3D finite element model has been developed. The comparison between the predictions from the presented semi-analytical and those from the 3D finite element models shows that the semi analytical model achieves very accurate predictions at a marginal computational time.
\end{abstract}

Keywords Elastic-Plastic, Finite Difference, FEM, Impact Force, Indentation, Transient Response, Abaqus

\section{Introduction}

The elastic-plastic impact behavior investigation is a prominent research topic. It is targeted by mechanical engineering researchers, in order to understand the relationship force-indentation of the contact. This is not an easy task because of the plastic deformation behavior inside the contact. The elastic-plastic transient response analysis of beam crashed by a projectile is necessary to study several phenomena such as the structural response and the local elastic-plastic contact behavior [1]. Several theoretical contact models have been used to study the relationship contact-force-indentation [2, 3], with the consideration of the rigid plastic idealization $[4,9]$. It is one of the simplifying hypothesis which neglects the elastic deformations compared to the plastic deformations whose purpose is to simplify the theoretical study. The elastic-plastic analyses are necessary to accurately study the transient impact response of structures.

Many elastic-plastic models have been developed to analyze the structural response, but they are limited. For a credible study, the deflection of the beam, the plasticity of the impacted area and the plasticity out-of-contact areas must be taken into account. Zhang et al [10] presented a hybrid, numerical-analytical model for elastic-plastic beam impact system with consideration of global elastic plastic deformation of the beam applied to analyze the transient impact response for low impact velocity. They found that the impact force response is influenced by impact-induced wave propagation and that the model is especially suitable for studying impact-induced wave effects. The maximum velocity used is $3.13 \mathrm{~m} / \mathrm{s}$, and they have clearly stated that with high velocity the plastic elastic behavior may appear in certain regions other than the contact region and that general analytical methods are not available. Wang et al [11] investigated theoretically and experimentally the applicability of different contact models on transverse repeated impacts of a sphere to an elastic-plastic beam. The investigations showed that for accumulated permanent indentation, Hertz [12] and MJG [13] contact models are in well correspendance with experimental results and for the contact time, Hertz model is in good agreement with experimental results according to low velocities.

The aim of this paper is to elaborate a semi-analytical model of beams crashed by a spherical projectile and to analyze the transversal impact. The stress and deformation of elastic-plastic contact are assumed to be close to the contact area, whose dimensions are small compared to the size of the contact bodies. The Stronge model [2] is used to calculate the contact force and to represent the elastic-plastic deformation behavior. The elastic-plastic 
transient behavior is influenced by the projectile impact velocity, which could occur in out-of-contact areas. The presented model is able to handle cases where plastic deformation is not confined to the impact point. A more general plasticity integration allowing to take into account the hardening is elaborated, The present model allows obtaining results of elastic plastic deformation especially equivalent plastic strain at integration point (PEEQ) in different zone, and the global deformation behavior of the beam according to low and high velocities.

\section{Problem Formulation}

Let us consider a simply supported elastic-perfectly plastic beam struck by a compact elastic-perfectly plastic sphere. The impact velocity is in the middle of the beam as shown in fig. 1 .

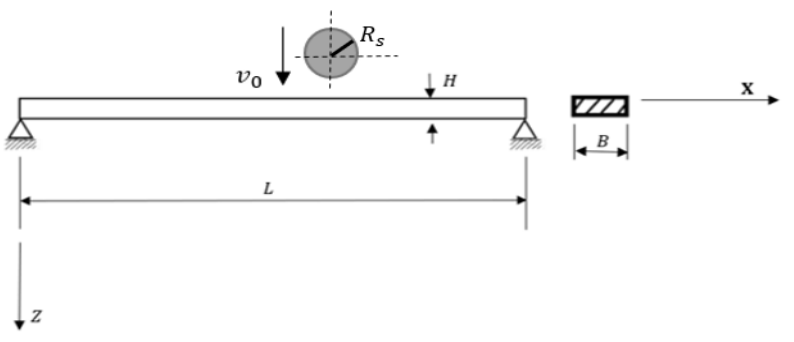

Figure 1. Impact of a sphere on simply supported beam

The equation of motion of the sphere is:

$$
m_{s} \ddot{w}_{S}=-F(t)+m_{s} g
$$

where $m_{s}$ and $w_{s}$ are respectively the mass and the displacement of the sphere, $F$ is the impact force.

The initial conditions of the sphere are:

$$
w_{s}(0)=0, \dot{w}_{s}(0)=v_{0}
$$

Among several phenomena caused by the impact, we could find the relationship contact force-indentation provided by the Stronge model. The indentation between the sphere and beam is

$$
\delta=w_{s}-w_{b}
$$

The displacement of the impacted point $w_{b}$ and of the sphere will be calculated in the same increment of time by the finite difference model.

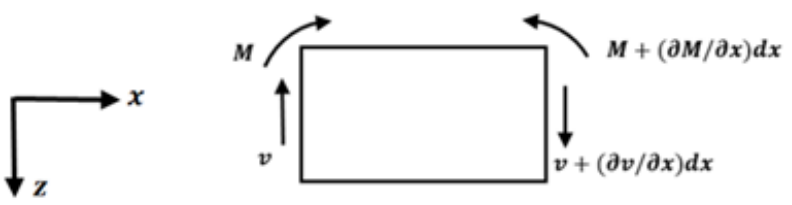

Figure 2. Elementary part of the beam

Figure 2 shows an elementary part of the beam, the axes $x$ and $z$ are respectively the axial and transverse direction.
The internal forces $F_{\text {int }}$ are the lateral shear force $\mathrm{v}$ and the bending moment $M$, the external force $F_{\text {ext }}$ is the distribution of the contact force $p(x, t)$ applied in the transverse direction. Without the damping consideration, the equation of motion of impacted beam is:

$$
\frac{\partial^{2} M_{b}}{\partial x^{2}}+p(x, t)=\rho_{b} A \frac{\partial^{2} w_{b}}{\partial t^{2}}
$$

where $\rho_{\mathrm{b}}$ and $A$ are respectively the mass density and the cross-section area of the beam.

$w_{b}$ is the transverse displacement of the beam.

It should be noted that the elastic perfectly plastic model is used in this study and the loading unloading contact phases are considered.

The following assumptions are adopted in the theoretical model for the motion of the beam:

- The effects of strain-rate are negligible

- Transverse shear effect is ignored

To investigate the transient impact behavior of the considered elastic-plastic beam, a semi analytical procedure based on the finite difference method in the axial direction and the Lobato integration rule in the thickness direction is firstly developed. For the sake of generality and comparison a 3D finite element method analysis is elaborated based on ABAQUS.

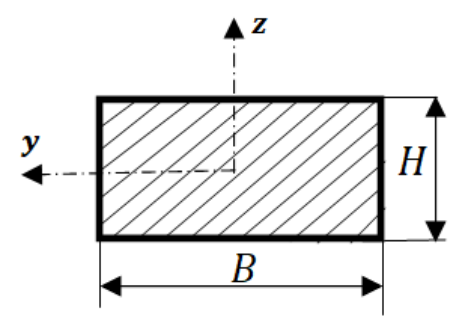

Figure 3. Cross section of the beam.

Figure 3 shows a cross section of the beam where the bending moment is calculated by the following integral:

$$
M=\int_{A} \sigma(z, t) z d A=B \int_{-H / 2}^{H / 2} z \sigma d z
$$

$\sigma(z, t)$ is the bending stress.

The equation of the motion of the beam can be written as follows:

$$
\rho_{b} A \ddot{w}=F_{\text {ext }}-F_{\text {int }}=R
$$

where $\mathrm{R}$ is the residual force.

The finite difference discretization of this equation leads to:

$$
\rho_{b} A \ddot{w}^{\mathrm{n}}=F_{\text {ext }}^{n}-F_{\text {int }}^{n}=R^{n}
$$

The internal force will be calculated by finite difference approach:

$$
F_{i n t}^{n}=\frac{\partial^{2} \mathrm{M}^{\mathrm{n}}}{\partial \mathrm{x}^{2}}=\frac{M_{n-1}-2 M_{n ?}+M_{n+1}}{\Delta \mathrm{x}^{2}}
$$


The solution is advanced using an explicit load stepping algorithm:

$$
\begin{gathered}
\dot{w}^{\mathrm{n}+\frac{1}{2}}=\dot{w}^{\mathrm{n}-\frac{1}{2}}+\ddot{w}^{\mathrm{n}-\frac{1}{2}} \Delta t \\
w^{n+1}=w^{n}+\Delta t \dot{w}^{n+\frac{1}{2}}
\end{gathered}
$$

$\ddot{w}^{\mathrm{n}}$ is computed from equation (7)

The initial conditions of the beam are:

$$
w(x, 0)=0, \dot{w}(x, 0)=0
$$

The boundary conditions of the beam are:

$$
\begin{aligned}
& w(0, t)=0, w(L, t)=0 \\
& M(0, t)=0, M(L, t)=0
\end{aligned}
$$

Due to the stability and accuracy of solutions in explicit temporal integration schemes, the time step length must satisfy the condition:

$$
\Delta t<\frac{\Delta x}{\sqrt{\frac{E_{b}}{\rho_{b}}}}
$$

\section{Contact Model Selection}

In fact whenever the impact velocity is slow, the contact area is small compared to the size of contact bodies. The concentration of contact stress and contact deformation is limited to the contact area. In the present analysis, the Hertz elastic contact model [12] and Stronge model [2] are used for the elastic loading and unloading phases.

The equivalent modulus of elasticity and radius are defined by:

$$
\frac{1}{E^{*}}=\frac{1-v_{s}^{2}}{E_{S}}+\frac{1-v_{b}^{2}}{E_{b}} ; \frac{1}{R^{*}}=\frac{1}{R_{S}}+\frac{1}{R_{b}}
$$

where $E^{*}, R^{*}$ are respectively the effective Young's modulus and the effective contact radius. $E_{s}, v_{s}$ are the Young's modulus and the Poisson's ratio of the sphere and $E_{b}, v_{b}$ the Young's modulus and the Poisson's ratio for the beam.

The radius of the beam $R_{b}=\infty$, therefore, the radius of the sphere $R_{S}=R^{*}$. is:

The relationship force-indentation in the elastic contact

$$
F=\frac{4}{3} E^{*} \sqrt{R^{*}} \delta^{\frac{3}{2}}
$$

\subsection{Loading Phase}

There are three sub-phases in the loading case, as listed in table 1. For spherical indentation, the elastic indentation at yielding is:

$$
\delta_{y}=\left(\frac{3 \Pi \vartheta_{y} \sigma_{y}}{4 E^{*}}\right)^{2} R^{*}
$$

$\vartheta_{y}=1.1$ and $\sigma_{y}=\sigma_{y b}$ the yielding stress of the beam.

The initiation of yielding at a point below the contact surface is caused by Hertz pressure. From this point, the plastically deformed region extends when the mean pressure increases above the yield pressure. However, the plastic deformation, which is progressing as contact pressure increases, the phenomenon below the contact surface continues until the most of the elastoplastic range. The incompressibility of the plastic deformation provokes a small quasi static elastic-plastic indentation of the surface. [2].

The indentation becomes perfectly plastic and begins at a contact radius when the plastic deformation develops outside the region beneath the contact.

\subsection{Unloading Phase}

The contact region during the unloading phase is considered elastic. The force-indentation relationship follows then Hertzian contact:

$$
F=\frac{4}{3} E^{*} \sqrt{R^{* e}}\left(\delta-\delta_{r}\right)^{1.5}
$$

The complet unloading from maximum indentation $\delta_{m}$ which is in the plastic deformation range causes a permanent indentation $\delta_{r}$ and a new unloaded curvature $R^{* e}$ of the contact area.

Stronge model [2] assumed a geometric similarity:

$$
\frac{\delta_{y}}{R^{*}}=\frac{\left(\delta_{m}-\delta_{r}\right)}{R^{* e}}
$$

The continuity of the contact force at the transition from the loading to unloading phase is a requirement for obtaining $\delta_{r}$ and $R^{* e}$. This condition is not valid when using the Stronge model [11].

To validate the condition and continue to use the Stronge model Wang et al [11] assumed that the initial force of the unloading is equal to the maximum contact force of the loading phase $F_{m}$ :

$$
F_{m}=\frac{4}{3} E^{*}\left(R^{* e}\right)^{0.5}\left(\delta_{m}-\delta_{r}\right)^{3 / 2}
$$

The substitution of equation (15) into equation (16) leads to the following expression of $\delta_{r}$ and $R^{* e}$ :

$$
R^{* e}=\sqrt{\frac{3}{4} \frac{F_{m}}{E^{*}}}\left(\frac{R^{*}}{\delta_{y}}\right)^{\frac{3}{4}}, \delta_{r}=\delta_{m}-\frac{\delta_{y} R^{* e}}{R^{*}}
$$


Table1. Formulas of elastic-plastic contact Stronge Model

\begin{tabular}{|c|c|c|c|c|}
\hline \multicolumn{5}{|c|}{ Stronge Model } \\
\hline Elastic Phase & Indentation at yield & Elastic-Plastic phase & Fully Plastic phase & Unloading Phase \\
\hline $\mathrm{F}=\frac{4}{3} \mathrm{E}^{*} \sqrt{\mathrm{R}^{*}} \delta^{\frac{3}{2}}$ & $\delta_{\mathrm{y}}=\left(\frac{3 \Pi \vartheta_{\mathrm{y}} \sigma_{\mathrm{y}}}{4 \mathrm{E}^{*}}\right)^{2} \mathrm{R}^{*}$ & $\mathrm{~F}=\mathrm{F}_{\mathrm{y}}\left(\frac{2 \delta}{\delta_{\mathrm{y}}}-1\right)\left(1+\frac{\ln \left(2 \delta / \delta_{\mathrm{y}}-1\right)}{3 \vartheta_{\mathrm{y}}}\right)$ & $\mathrm{F}=2.8 \mathrm{~F}_{\mathrm{y}}\left(2 \delta / \delta_{\mathrm{y}}-1\right) / \vartheta_{\mathrm{y}}$ & $\begin{array}{c}\mathrm{F}=\frac{4}{3} E^{*}\left(R^{* e}\right)^{0.5}\left(\delta-\delta_{r}\right)^{3 / 2} \\
\delta_{r}=\delta_{m}-\frac{\delta_{y} R^{* e}}{R^{*}}\end{array}$ \\
\hline & $\vartheta_{\mathrm{y}}=1.1$ & $\mathrm{~F}_{\mathrm{y}}=\frac{4}{3} \mathrm{E}^{*} \sqrt{\mathrm{R}^{*}} \delta_{\mathrm{y}}^{\frac{3}{2}}$ & & $R^{* e}=\sqrt{\frac{3}{4} \frac{F_{m}}{E^{*}}\left(\frac{R^{*}}{\delta_{y}}\right)^{\frac{3}{4}}}$ \\
\hline & & & & $\delta_{r}=\delta_{m}-\frac{\delta_{y} R^{* e}}{R^{*}}$ \\
\hline
\end{tabular}

\section{Results and Discussion}

Transient impact behavior of elastoplastic beams is investigated based on the elaborated semi-analytical formulation and on the 3D finite element method using ABAQUS. The used geometrical and material properties are given in tables 2 and 3.

Table 2. Dimensional detail of the beam and sphere

\begin{tabular}{|c|c|}
\hline$H$. Depth & $27.8 \mathrm{~mm}$ \\
\hline$B$. Width & $60 \mathrm{~mm}$ \\
\hline$L$. Length & $780 \mathrm{~mm}$ \\
\hline$R_{s}$. Radius of the sphere & $35 \mathrm{~mm}$ \\
\hline$v_{0}$. Impact velocity & $0.99 \mathrm{~m} / \mathrm{s}$ \\
\hline
\end{tabular}

Table 3. Mechanical material properties of the beam and sphere

\begin{tabular}{|l|c|l|c|}
\hline \multicolumn{2}{|c|}{ Sphere Gr 15 } & \multicolumn{2}{c|}{ Beam Q345 } \\
\hline$\rho_{s}$ & $7800 \mathrm{Kg} / \mathrm{m}^{3}$ & $\rho_{b}$ & $7800 \mathrm{Kg} / \mathrm{m}^{3}$ \\
\hline$m_{s}$ & $1.4 \mathrm{Kg}$ & $m_{b}$ & $10.929 \mathrm{Kg}$ \\
\hline$E_{s}$ & $208000 \mathrm{Mpa}$ & $E_{b}$ & $210000 \mathrm{Mpa}$ \\
\hline$\sigma_{y s}$ & $2550 \mathrm{Mpa}$ & $\sigma_{y b}$ & $345 \mathrm{Mpa}$ \\
\hline$v_{s}$ & 0.3 & $v_{b}$ & 0.3 \\
\hline
\end{tabular}

Figure 4 shows a quarter FE discretization of the impact problem used to simplify the modeling and to reduce the CPU time. The mesh size of the high-stress contact zone was refined, the smallest size being $0.2 \mathrm{~mm}$. The farthest contact area has been meshed with coarse mesh.

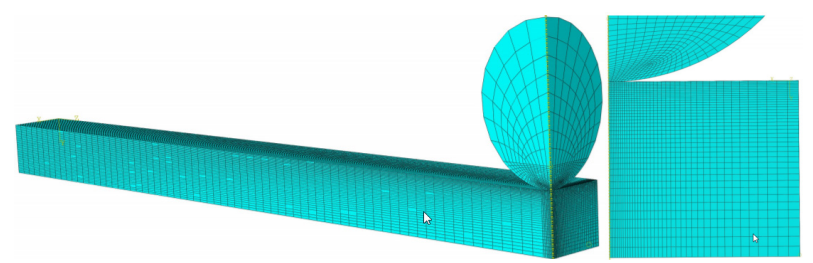

Figure 4. 3D FE Model modeled by ABAQUS

Figure 5 (a) shows the impact force evolution with the time reduced to $0.3 \mathrm{~ms}$, by superimposing the semi analytical, 3D FE and reference results. The semi analytical solution is clearly in good agreement with the finite element solution obtained by ABAQUS software with a $2.4 \%$ error. The reference results from Zhang et al. [10] are offset to the finite element solution results with a $6.9 \%$ error. Our model is therefore in a good agreement with FE results compared to the reference model, This may be explained by the fact that we have used a finite difference discretization in the longitudinal direction and an integration through the thickness of the beam provided by Lobato rule, whereas Zhang et al [10] have used the finite difference discretization in the longitudinal direction and more throught the thickness of the beam.

Figure 5 (b) shows the superimposed semi analytical and 3D FE displacement evolution with the time reduced to 0.4 ms. A slight discrepancy in the displacement evolution between the semi analytical and FE results, is resulted proving that a good correlation was achieved between the semi analytical and the 3D FE displacement evolution.
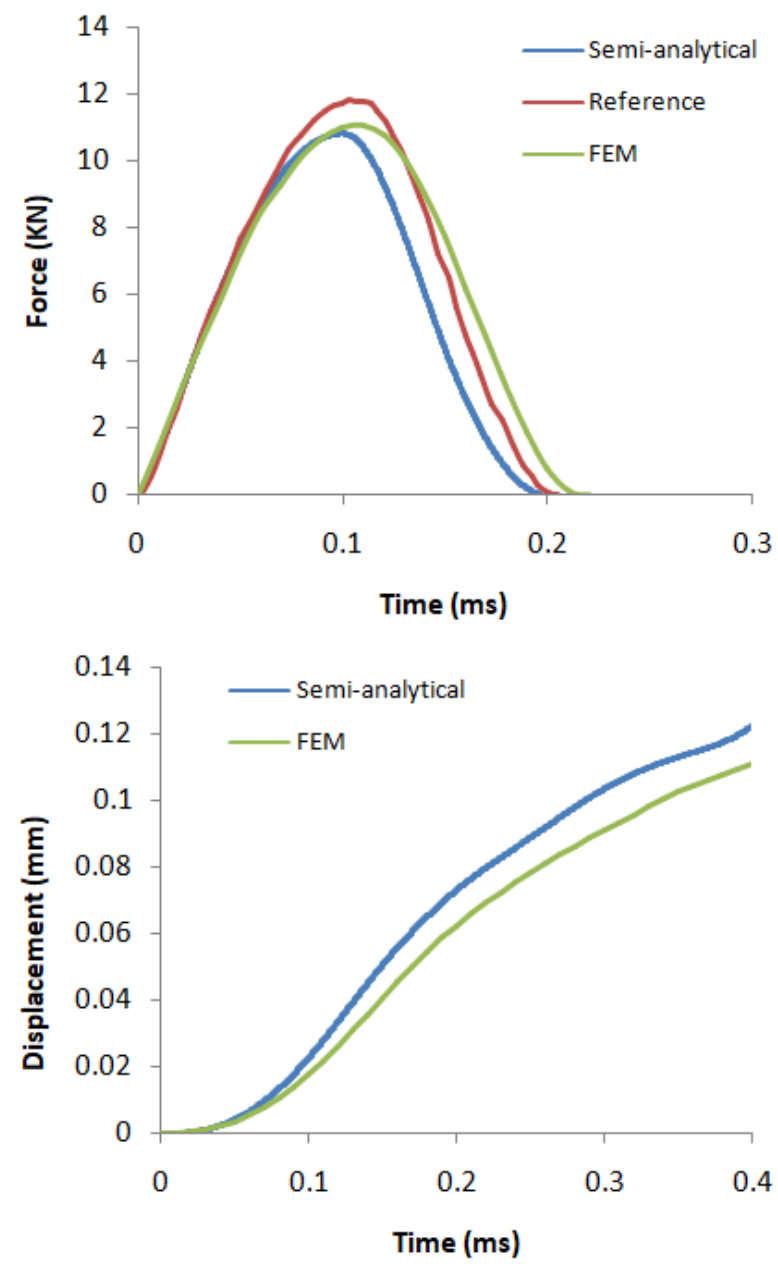

Figure 5. (a) Impact force evolution (b) Displacement evolution of the beam 
Figure 6 demonstrates that the number of finite difference points $\mathrm{N}$ through the longitudinal direction, and the number of integration points $\mathrm{P}$ through the thickness have a deep influence on the results convergence. The convergence with respect to discretization points numbers is demonstrated. A minimum of $\mathrm{N}=601$ and $\mathrm{P}=2$ is required for reaching the accurate FEM results. It should be noted that Lobato rule is used for the integration through the thikness.
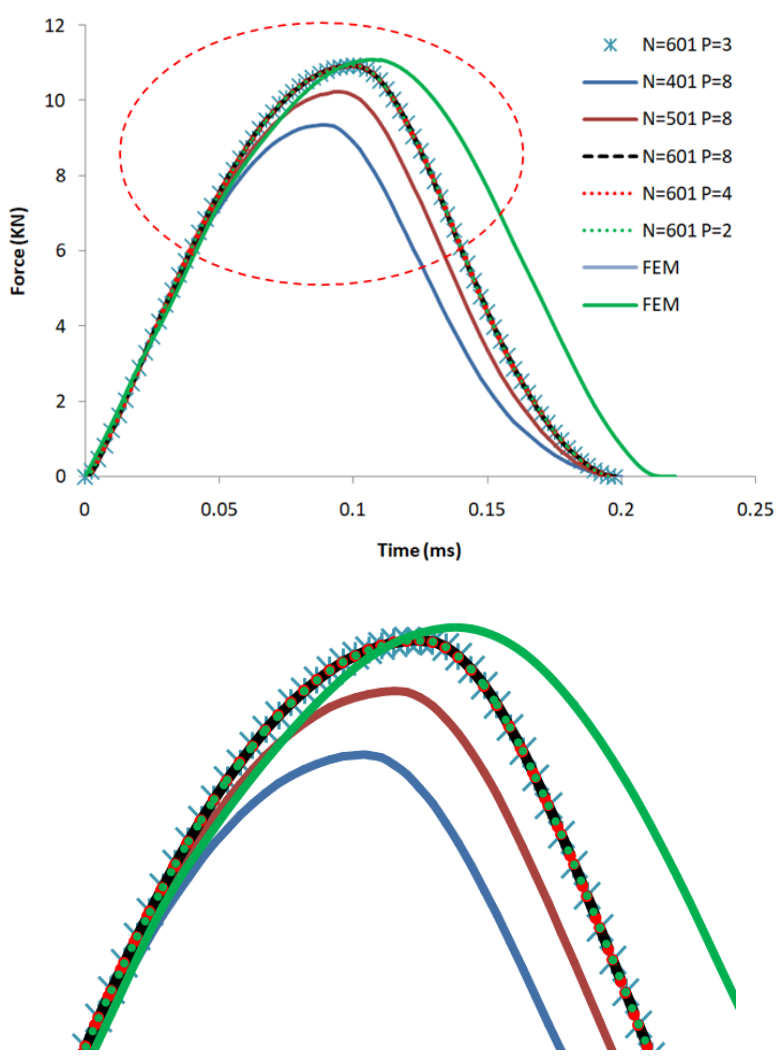

Figure 6. Effect of the number of finite difference points $\mathrm{N}$ and the number of integration points $\mathrm{P}$ on the results convergence
Figure 7 shows the impact indentation force relationship by low and intermadiate velocities, it is completed, by continuity at the transition point from the loading phase to the unloading phase. By increasing the impact velocity the indentation increases with a larger permanent indentation.

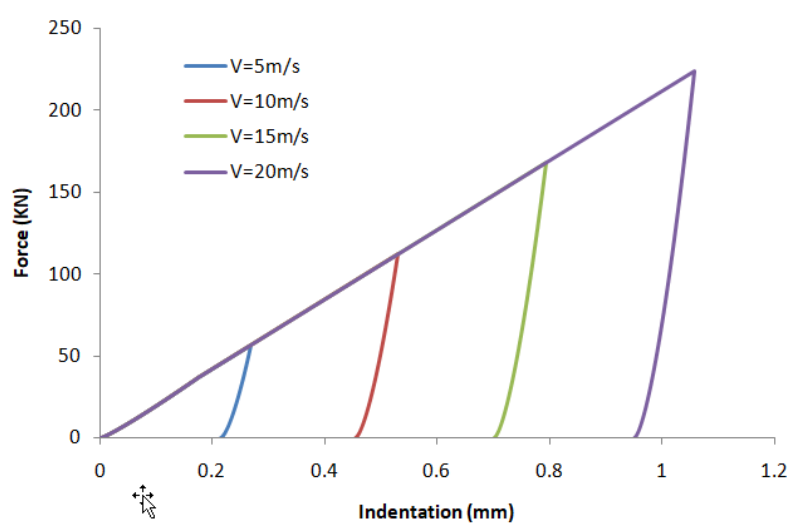

Figure 7. Impact force-Indentation relationship with low and high velocities

The contact time as a function of impact velocity of the sphere is demonstrated in figure 8. The contact time decreases by increasing the impact velocity, notice that our model based on the stronge theory reaches the finite element solution obtained by ABAQUS software as well as with the experimental measurements from Wang et al [11]. However, the theoretical solutions from Wang et al [11] based on the Stronge theory are below the experimental meassurements. This may be caused by the model type. We are quoting that Wang et al [11] have modeled the beam motion as a spring-mass to simplify the problem, whereas we have modeled the beam motion using the finit difference methode by discretization in the longitudinal direction and numerical integration through the thickness of the beam provided by Lobato rule.

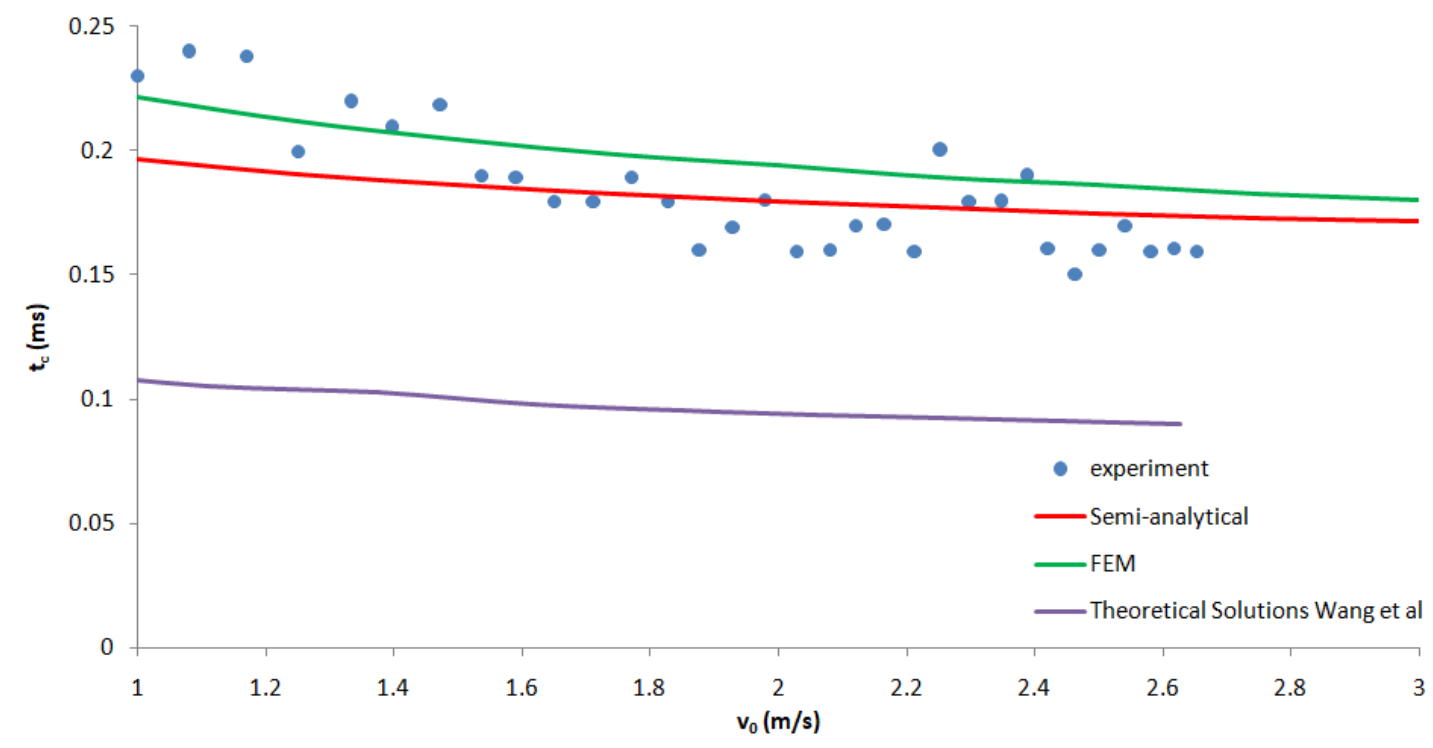

Figure 8. Contact time of the semi-analytical solutions experiments and FEM 

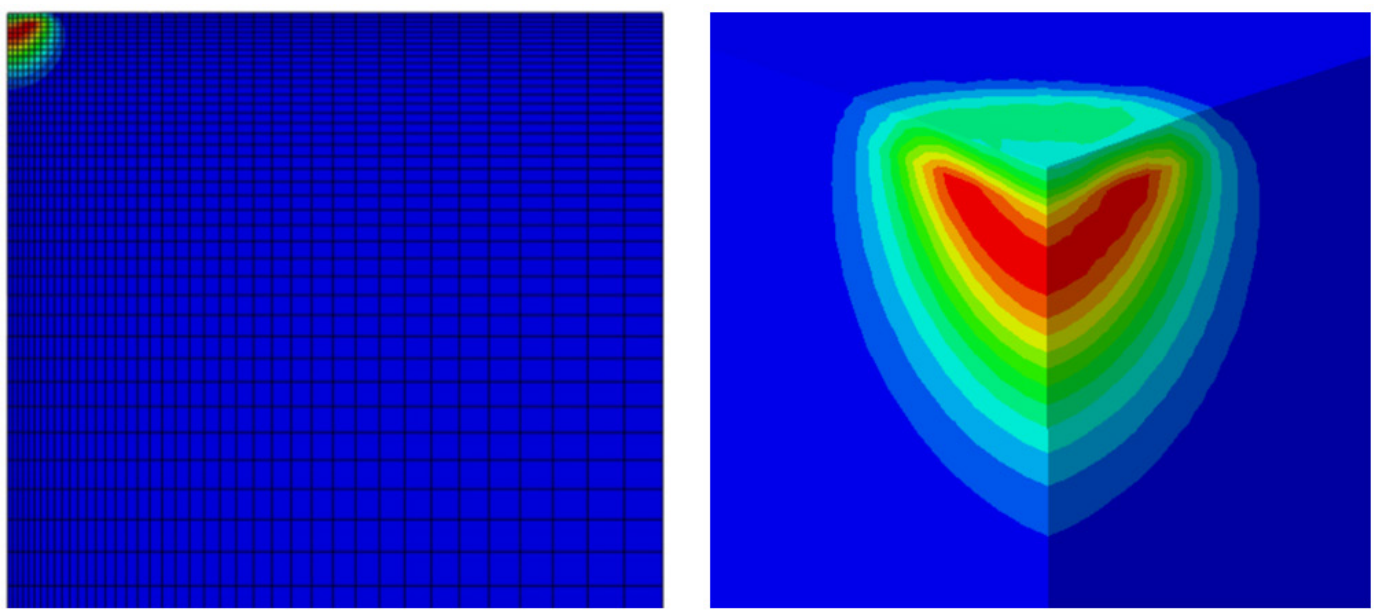

Figure 9. Plastic Deformation result of 3D FE with $v_{0}=0.99 \mathrm{~m} / \mathrm{s}$

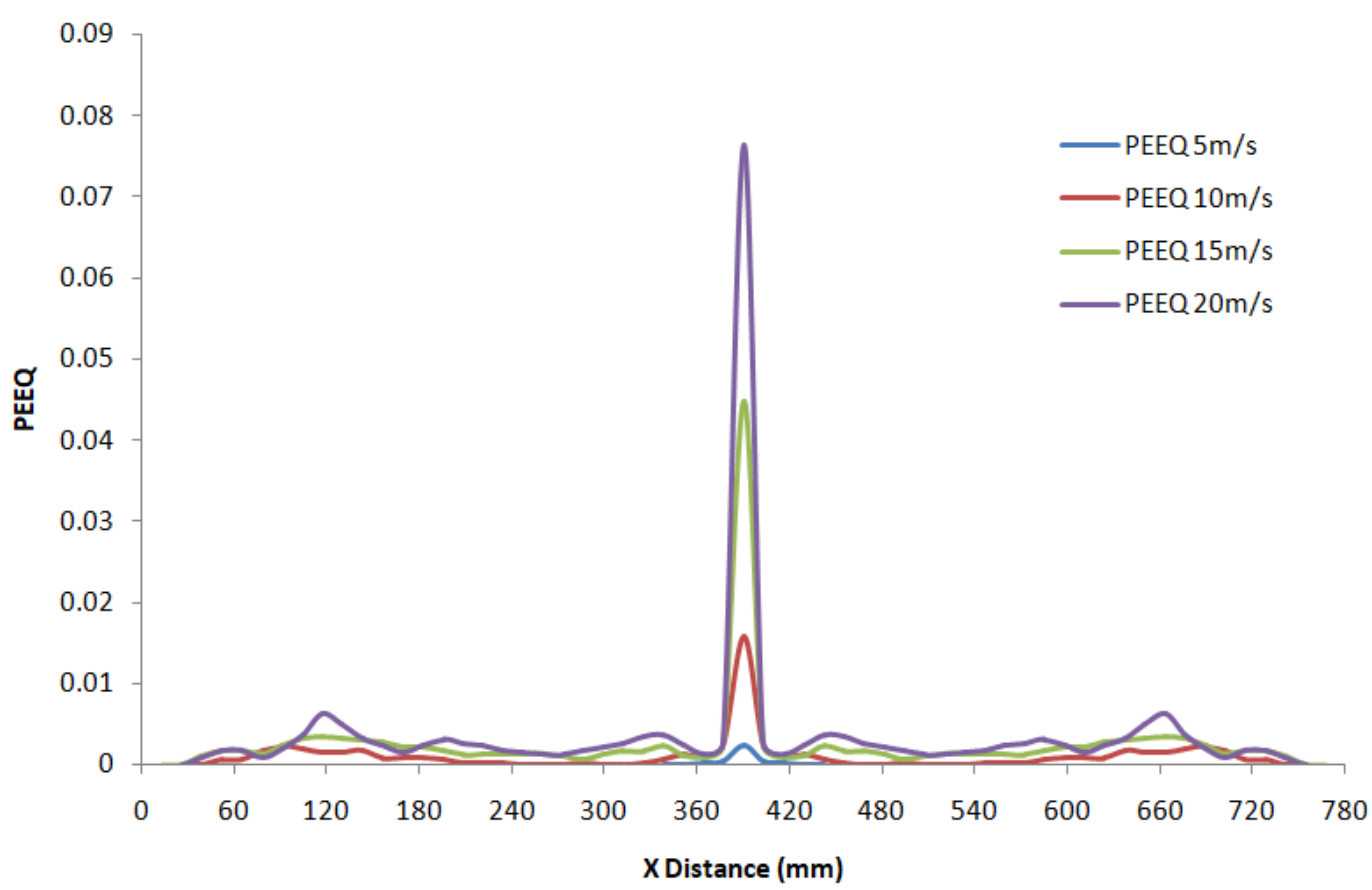

Figure 10. Equivalent Plastic Strain prediction from the semi-analytical model with low and high velocities

The plastic deformation area, at a distance of $1.52 \mathrm{~mm}$ from the center of the beam is shown in figure 9. It is observed that the plastic deformation remains below the contact zone with an impact velocity $v_{0}=0.99 \mathrm{~m} / \mathrm{s}$.

The plastic peak of the beam for low and high velocities from 5 to $20 \mathrm{~m} / \mathrm{s}$ is presented in figure 10 . For impact velocity from 0 to $5 \mathrm{~m} / \mathrm{s}$, the highest plastic peak is concentrated under the point of impact with no plastic deformation throughout the beam. For $10 \mathrm{~m} / \mathrm{s}$ of impact velocity we have noticed that the maximum plastic peak value is concentrated under the point of impact with plastic deformation of less than $0.2 \%$ throughout the beam. For 15 to $20 \mathrm{~m} / \mathrm{s}$ of impact velocity with plastic deformation of less than $0.2 \%$ throughout the beam we found that the plastic peak is not only concentrated under the point of impact but it is presented with a value larger than $0.2 \%$ on the other points of the beam.

With a low velocity it is found that the plastic deformation is limited to the point of impact, while a high impact velocity causes plastic deformation by bending elsewhere than under the impact point.

\section{Conclusions}

In this paper, we presented a semi-analytical model combining finite difference method with the Hertz contact theory and the Stronge model. The considered impact problem of elastic plastic beams impacted by a low velocity projectile was numerically solved. The model results are compared with the 3D FE model. It was demonstrated that the model is credible and can analyze the 
impact response under various impact velouties. It is effective and very fast in comparison with the FE model. It was shown that the finite difference discretization size and the number of integration points through the tickness have an influence on the results convergence. The plastic deformation is influenced by the impact velocity. We have noticed that a high velocity causes plastic deformation by bending.

\section{REFERENCES}

[1] A.S. Yigit, A.P. Christoforou, M.A.Majeed. A nonlinear visco-elastoplastic impact model and the coefficient of restitution, Nonlinear Dyn, 66 509-521. 2011

[2] W.J. Stronge, Impact Mechanics, Cambridge University Press, London, 2000.

[3] M. Machado, P. Moreira, P.Flores, H.M. Lankarani, Compliant contact force models in multibody dynamics: evolution of the

[4] W.J. Stronge, T. Yu, Dynamic Models for Structural Plasticity, Springer, New York, 1993.

[5] J. Lellep, K. Torn, Shear and bending response of a rigid-plastic beam subjected to impulsive loading, Int. J. Impact Eng. 31 (2005) 1081-1105.

[6] E. Lee, P.S. Symonds, Large plastic deformations of beams under transverse impact, ASME J. Appl. Mech. 19 (1952) 308-314.

[7] S.H. Ghaderi, K. Hokamoto, M. Fujita, Analysis of stationary deformation behavior of a semi-infinite rigid-perfect plastic beam subjected to moving distributed loads of finite width, Int. J. Impact Eng. 36 (2009) 115-121.

[8] N. Jones, Structural Impact, Cambridge University Press, Cambridge, 2012

[9] L. Zhu, D. Faulkner, Damage estimate for plating of ships and platforms under repeated impacts, Marine Structures 9 (7) (1996) 697-720.

[10] L. Zhang, X. Yin, J.Yang, H.Wang, Q.Deng, B.Yu, Q.Hao , H.Ding, X. Qi , T.Jin, X.Dong, Transient impact response analysis of an elastic-plastic beam, Applied Mathematical Modelling, 55 616-636, 2018.

[11] H. Wang , X. Yin , X. Qi , Q. Deg , B. Yu , Q. Hao, Experimental and theoretical analysis of the elastic-plastic normal repeated impacts of a sphere on a beam , Int. J. Solids Structures, 109 131-142, 2017.

[12] H. Hertz, Über die Berührung fester elastischerKörper, Journal für die reine und angewandteMathematik, 92 156-171, 1881.

[13] H.Ghaednia, D.B. Marghitu, R.L. Jackson, 2014. Predicting the permanent deformation after the impact of a rod with a flat surface. J. Tribol. 137 (1), 011403 